\title{
Constraining the internal dynamics of stellar systems using the NMAGIC particle code
}

\author{
Flavio De Lorenzi ${ }^{1,2} \dagger$, Ortwin Gerhard ${ }^{1}$, Victor P. Debattista ${ }^{3}$, \\ Niranjan Sambhus ${ }^{2}$, L. Coccato ${ }^{1,4}$, M. Arnaboldi ${ }^{5,6}$, M. Capaccioli ${ }^{7}$, \\ N. G. Douglas ${ }^{4}$, K. C. Freeman ${ }^{8}$, K. Kuijken ${ }^{9,4}$, M. R. Merrifield ${ }^{10}$, \\ N. R. Napolitano ${ }^{7}$, E. Noordermeer ${ }^{10}$ and A. J. Romanowsky ${ }^{11}$ \\ ${ }^{1}$ Max-Planck-Institut für Ex. Physik, Giessenbachstraße, D-85741 Garching, Germany \\ ${ }^{2}$ Astron. Institut, Universität Basel, Venusstrasse 7, Binningen, CH-4102, Switzerland \\ ${ }^{3}$ Centre for Astrophysics, University of Central Lancashire, Preston, UK PR1 2HE \\ ${ }^{4}$ Kapteyn Astronomical Institute, University of Groningen, the Netherlands \\ ${ }^{5}$ ESO, Karl-Schwarzschild-Str. 2, D-85748 Garching Germany \\ ${ }^{6}$ INAF, Observatory of Turin, Strada Osservatorio 20, 10025 Pino Torinese, Italy \\ ${ }^{7}$ Osservatorio di Capodimonte, Naples, Italy \\ ${ }^{8}$ Research School of Astronomy \& Astrophysics, ANU, Canberra, Australia \\ ${ }^{9}$ University of Leiden, The Netherlands \\ ${ }^{10}$ School of Physics \& Astronomy, University of Nottingham, UK \\ ${ }^{11}$ Departamento de Física, Universidad de Concepsión, Casilla 160-C, Concepción, Chile
}

\begin{abstract}
NMAGIC is a parallel implementation of our made-to-measure ( $\left.\chi^{2} \mathrm{M} 2 \mathrm{M}\right)$ algorithm for constructing N-particle models of stellar systems from observational data, which extends earlier ideas by Syer \& Tremaine (1996). The $\chi^{2}$ M2M algorithm properly accounts for observational errors, is flexible, and can be applied to various systems and geometries. We show its ability to reproduce the internal dynamics of an oblate isotropic rotator model and report on the modeling of the dark matter (DM) halo of NGC 3379 combining SAURON and PN.S kinematic data. The $\chi^{2} \mathrm{M} 2 \mathrm{M}$ algorithm is practical, reliable and can be applied to various dynamical systems without symmetry restrictions. We conclude that $\chi^{2} \mathrm{M} 2 \mathrm{M}$ holds great promise for unraveling the internal dynamics of bulges.
\end{abstract}

Keywords. galaxies: elliptical and lenticular, $\mathrm{cD}$, galaxies: kinematics and dynamics, galaxies: individual: NGC 3379, methods: numerical, methods: n-body simulations

\section{Introduction}

Syer \& Tremaine (1996) invented a method to construct "made-to-measure" (M2M) particle models of stellar systems. The M2M method works by gradually changing the weights of the particles with time, until the model reproduces a set of observational constraints. Bissantz et al. (2004) made a first practical application of the M2M method and modeled the face-on density distribution of the Milky Way. Their model was able to match the microlensing event timescale distribution towards the galactic bulge. De Lorenzi et al. (2007) improved the M2M algorithm to account for observational errors and implemented their $\chi^{2} \mathrm{M} 2 \mathrm{M}$ algorithm in a parallel code NMAGIC. Here, after describing some tests of the method, we report on one of the first applications of NMAGIC, modeling the halo of the elliptical galaxy NGC 3379.

$\dagger$ email: lorenzi@mpe.mpg.de 

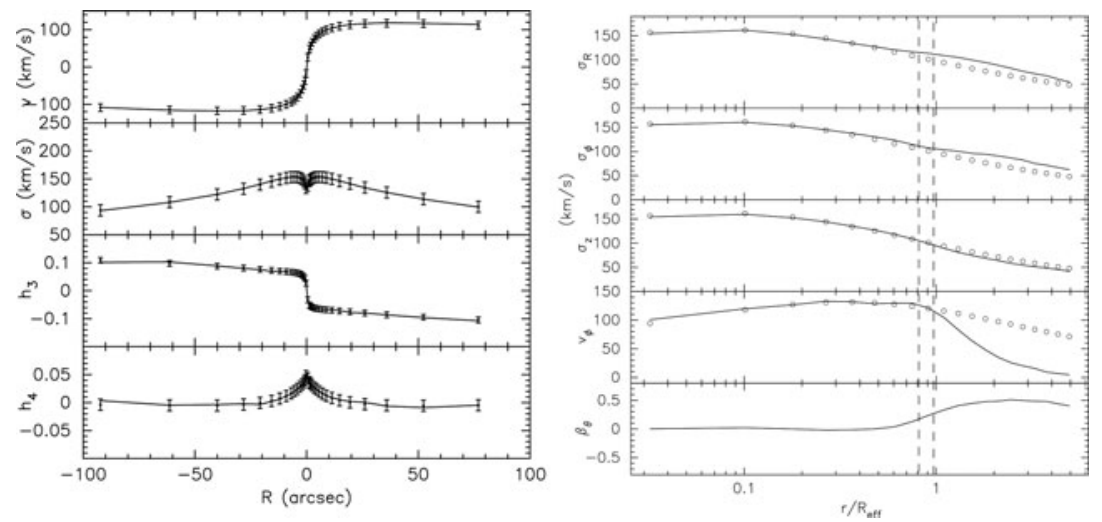

Figure 1. (a) Left: NMAGIC model fit to the isotropic rotator mock kinematic data along the major axis. The data points show the target data and the lines represents the model. (b) Right: Internal velocity moments and anisotropy parameter $\beta_{\theta}=1-\sigma_{\theta}^{2} / \sigma_{r}^{2}$ in the meridional plane for the self-consistent isotropic rotator model. The data points represent the input model and the lines correspond to the final particle model. The dashed lines indicate the extent of the data on both sides of the major axis.

\section{Testing NMAGIC with isotropic rotator models}

We test our modeling with an axisymmetric, isotropic rotator model with known intrinsic properties. We have chosen to describe the luminosity density of the mock galaxy by one of the flattened $\gamma$-models of Dehnen \& Gerhard (1994):

$$
j(m)=\frac{(3-\gamma) L}{4 \pi q} \frac{a}{m^{\gamma}(m+a)^{4-\gamma}},
$$

where $L$ and $a$ are the total light and scale radius, $m^{2}=R^{2}+(z / q)^{2}$, with the flattening $q=0.7, \gamma=1.5, L=2 \times 10^{10} L_{\odot, R}$ and $a=2.5 \mathrm{kpc}\left(a \approx 49^{\prime \prime}\right.$ for the adopted distance $D=10.5 \mathrm{M} p c)$. We further assume that the galaxy is seen under $i=80^{\circ}$. For this target model, we compute photometric and kinematic observables, which are then used as constraints in building the particle model with NMAGIC. The photometric observables are given by a spherical harmonics decomposition of the luminosity density given in equation 2.1. The target kinematics are computed from internal velocity moments obeying higher-order Jeans equations (Magorrian \& Binney 1994) in the self-consistent potential generated by the density, assuming a mass-to-light ratio $\Upsilon=5$.

We construct a self-consistent particle model in a two step process. First, we start with spherical initial conditions made from distribution function (Debattista \& Sellwood 2000) and evolve it using NMAGIC to generate a self-consistent particle realization with the desired luminosity distribution ( $\gamma$-particle model), fitting only the luminosity constraints. We then switch retrograde particles of the $\gamma$-particle model with a probability

$$
p\left(L_{z}\right)=p_{0} \frac{L_{z}^{2}}{L_{z}^{2}+L_{0}^{2}},
$$

where $p_{0}=0.3$ and $L_{0}=0.02$ given in internal units. We then use the rotating, anisotropic $\gamma$-particle model as a starting point for a self-consistent model fit to the photometric and kinematic constraints. Figure 1 shows the fit to the kinematic data achieved by the final particle model, and compares the intrinsic velocity moments of the input model with those of the particle model. The input model is well reproduced within the constraint region. 

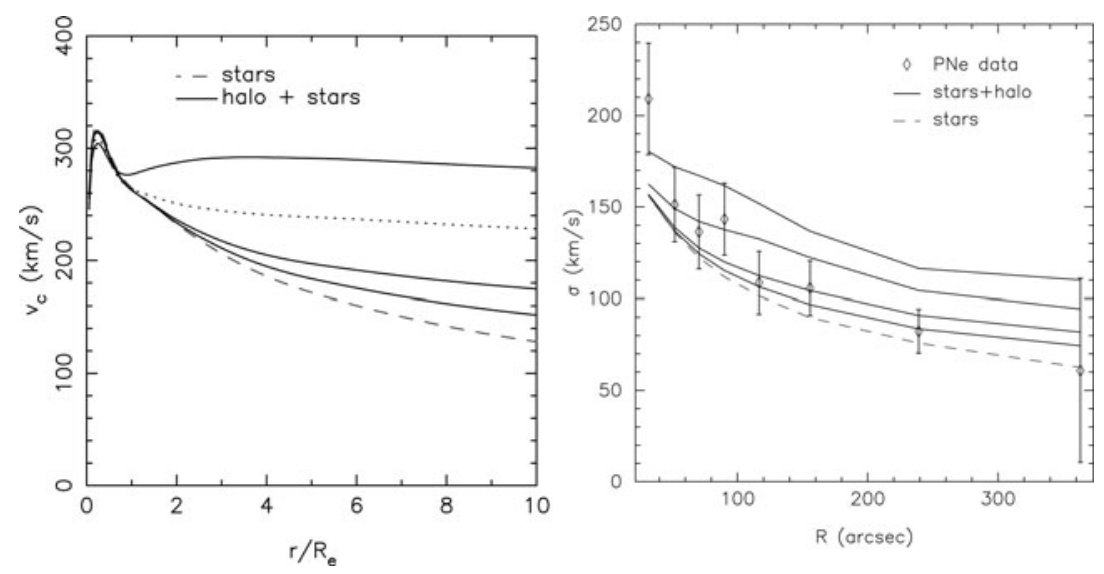

Figure 2. (a) Left: Circular velocity curves for mass models consisting of the stellar mass distribution of NGC 3379 plus various logarithmic DM halo profiles. The self-consistent model is shown by the dashed line. (b) Right: PN.S velocity dispersion data of NGC 3379 with superimposed spherical NMAGIC fits using the mass models of the left panel.

\section{NGC 3379}

We now use NMAGIC to construct spherical and axisymmetric dynamical models of NGC 3379, the strongest case of Romanowsky et al. (2003) for a diffuse DM halo. NGC 3379 is an intermediate luminosity E1 galaxy with distance $D=9.8 \mathrm{Mpc}$ and effective radius $R_{e}=47^{\prime \prime}$. We generate NMAGIC models including various DM halos as illustrated by the circular velocity curves in the left panel of Figure 2. We constrain the models using wide field B-band photometry of Capaccioli et al. (1990) combined with the HST V-band observations of the inner 10" of Gebhardt et al. (2000), SAURON integral field kinematics from Shapiro et al. (2006), and PN.S kinematic data from Douglas et al. (2007).

\subsection{Spherical models}

The right panel of Figure 2 shows the PN.S velocity dispersion data with superimposed spherical NMAGIC fits using the mass models shown in the left panel of Figure 2. The spherical models fit the combined data only in the presence of a diffuse DM halo.

\subsection{Oblate models}

There is some evidence that NGC 3379 is non-spherical (e.g. Statler 2001, and the cold ring in $\sigma$ of the SAURON data shown in Figure 3 at $\left.R \approx 15^{\prime \prime}\right)$. Therefore, we construct an axisymmetric dynamical model, assuming an inclination $i=40^{\circ}$ and including the DM halo indicated by the dotted line in the left panel of Figure 2. Figure 3 shows a comparison of the model with the SAURON and PN.S data. The axisymmetric model in a massive DM halo provides a viable fit to the data. It works by increasing $\sigma_{R} / \sigma_{\phi}$ and by decreasing $\sigma_{z}$, preferentially flattening the outer galaxy.

\section{Conclusion}

As a test of our modeling, we have constructed NMAGIC dynamical models of an isotropic rotator mock galaxy and showed that the internal velocity moments of the input system are well reproduced by the particle model. After this we have constructed spherical and axisymmetric dynamical models for NGC 3379, including different DM halos. The PNe velocity dispersion profile is consistent with a wide range of DM halos, and is not in contradiction with $\Lambda \mathrm{CDM}$. A similar conclusion was obtained in work not 


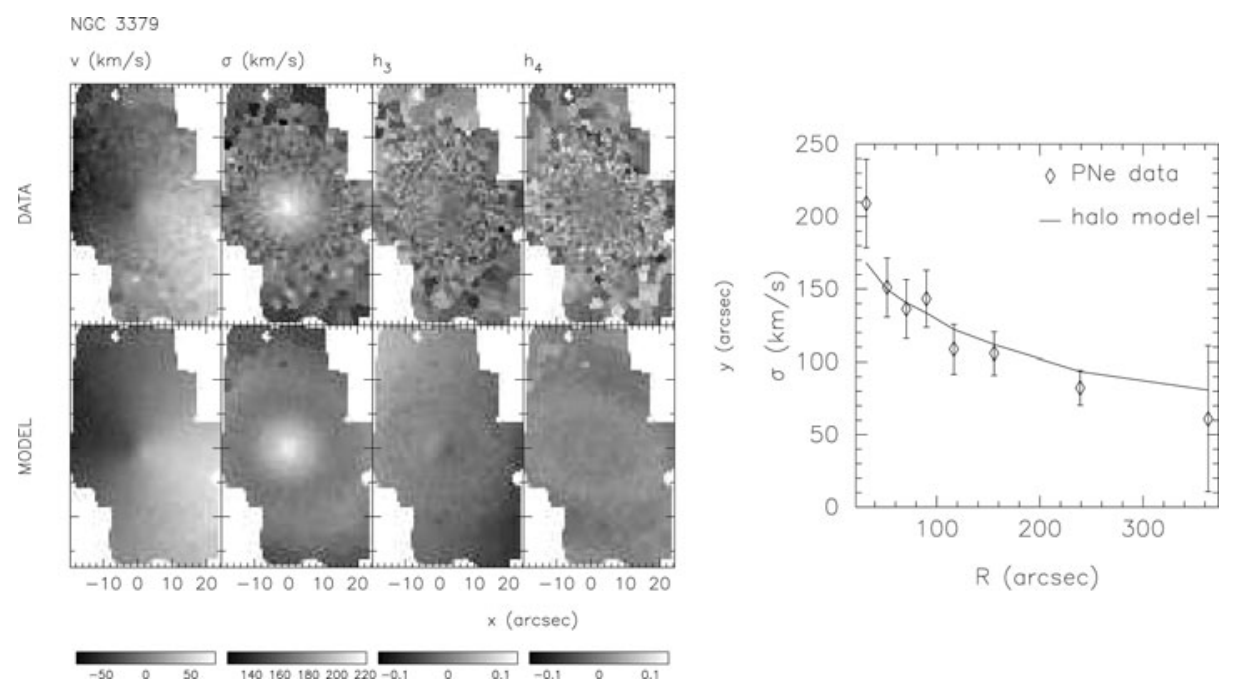

Figure 3. (a) Left: The top panels show the SAURON kinematic data for NGC 3379 and the bottom panels show the axisymmetric particle model fit. (b) Right: PNe velocity dispersion data of NGC 3379 with superimposed axisymmetric model. The NMAGIC fit was generated using the mass model indicated by the dotted line in the left panel of Figure 2.

reported here for the nearly edge-on elliptical galaxy NGC 4697 (see Méndez et al. 2001 and De Lorenzi et al. 2008).

\section{References}

Bissantz, N., Debattista, V. P., \& Gerhard, O. 2004, ApJ 601, L155

Capaccioli, M., Held, E. V., Lorenz, H., \& Vietri, M. 1990, AJ 99, 1813

Debattista, V. P. \& Sellwood, J. A. 2000, ApJ 543, 704

Dehnen, W. \& Gerhard, O. E. 1994, MNRAS 268, 1019

De Lorenzi, F., Debattista, V. P., Gerhard, O., \& Sambhus, N. 2007, MNRAS 376, 71

De Lorenzi, F., Gerhard, O., Saglia, R. P., Sambhus, N., Debattista, V. P., Panella, M., \& Méndez, R. H. 2008, submitted to MNRAS

Douglas, N. G., Napolitano, N. R., Romanowsky, A. J., Coccato, L., Kuijken, K., Merrifield, M. R., Arnaboldi, M., Gerhard, O., Freeman, K. C., Merrett, H. R., Noordermeer, E., \& Capaccioli, M. 2007, ApJ 664, 257

Gebhardt, K., Richstone, D., Kormendy, J., Lauer, T. R., Ajhar, E. A., Bender, R., Dressler, A., Faber, S. M., Grillmair, C., Magorrian, J., \& Tremaine, S. 2000, AJ 119, 1157

Magorrian, J. \& Binney, J. 1994, MNRAS 271, 949

Méndez, R. H., Riffeser, A., Kudritzki, R.-P., Matthias, M., Freeman, K. C., Arnaboldi, M., Capaccioli, M., \& Gerhard, O. E. 2001, ApJ 563, 135

Romanowsky, A. J., Douglas, N. G., Arnaboldi, M., Kuijken, K., Merrifield, M. R., Napolitano, N. R., Capaccioli, M., \& Freeman, K. C. 2003, Science 301, 1696

Shapiro, K. L., Cappellari, M., de Zeeuw, T., McDermid, R. M., Gebhardt, K., van den Bosch, R. C. E., \& Statler, T. S. 2006, MNRAS 370, 559

Statler, T. S. 2001, AJ 121, 244

Syer, D. \& Tremaine, S. 1996, MNRAS 282, 223 